\title{
Dynamic Wireless Energy Harvesting and Optimal Distribution in Multipair DF Relay Network with Nonlinear Energy Conversion Model
}

\author{
Syed Tariq Shah $\left(\mathbb{D},{ }^{1,2}\right.$ Daniel B. da Costa $\mathbb{D}^{\mathbb{2}},{ }^{3}$ Kae Won Choi $\mathbb{D}^{1},{ }^{1}$ and Min Young Chung $\mathbb{I D}^{1}$ \\ ${ }^{1}$ Department of Electrical and Computer Engineering, Sungkyunkwan University, 2066 Seobu-ro, \\ Jangan-gu, Suwon, Gyeonggi-do 16419, Republic of Korea \\ ${ }^{2}$ Department of Telecommunication Engineering, FICT, Balochistan University of Information Technology, \\ Engineering and Management Sciences, Pakistan \\ ${ }^{3}$ Department of Computer Engineering, Federal University of Ceará, Sobral, CE, Brazil \\ Correspondence should be addressed to Min Young Chung; mychung@skku.edu
}

Received 14 February 2018; Revised 13 July 2018; Accepted 24 July 2018; Published 6 August 2018

Academic Editor: Enrico Natalizio

Copyright (c) 2018 Syed Tariq Shah et al. This is an open access article distributed under the Creative Commons Attribution License, which permits unrestricted use, distribution, and reproduction in any medium, provided the original work is properly cited.

Wireless energy harvesting has emerged as an efficient solution to prolong the lifetime of wireless networks composed of energyconstrained nodes. In this paper, we consider a multipoint-to-multipoint relay network, where multiple source nodes communicate with their respective destination nodes via intermediate energy-constrained decode-and-forward (DF) relay. The performance of two different transmission modes, namely, delay tolerant and delay nontolerant, is studied. Based on power-splitting relaying protocol (PSR), optimal energy harvesting and distribution schemes for both transmission modes are provided. In addition, for more realistic and practical analysis, we consider a nonlinear energy conversion model for energy harvesting at the relay node. Our numerical results provide useful insights into different system parameters of a nonlinear energy harvesting-based multipair DF relay network.

\section{Introduction}

Energy harvesting via radio-frequency (RF) signals has emerged as a groundbreaking technique to prolong the network lifetime. The idea is to extend the lifetime of the network via wireless energy harvesting instead of replacing their batteries or recharging the devices through conventional methods [1-3]. Although other ambient energy harvesting methods such as thermoelectric effects, solar, vibrations, and the wind can also be used to recharge the batteries $[4,5]$, these conventional techniques are not very reliable and highly variable [6]. From the perspective of RF energy harvesting, the main advantage is that RF signals can simultaneously carry both information and energy. Thus, the energy-constrained nodes in the network can scavenge energy and process the information at the same time $[7,8]$. Note that, in a wireless energy harvesting enabled network, the nodes can harvest energy from both a dedicated RF source and an ambient RF source.

The idea of wireless energy harvesting offers a practical solution to extend the lifetime of energy constrained networks and also improve communication reliability. Due to these features, recent research works have widely studied its use in state-of-the-art next-generation technologies such as machine-to-machine communications (M2M), Internet of Things (IoT), MIMO, and 5G cellular networks [9-12]. Moreover, it is also well-known that relays can extend the coverage, improve quality-of-service (QoS), and improve capacity of networks by dividing the direct source-to-destination communication channel into two appropriate source-to-relay and relay-to-destination communication paths [13]. In conventional relay networks, relay node uses its own battery power to forward the information received from the source node. However, in the case of energy-constrained relay nodes, the 
network lifetime is significantly compromised. Luckily, recent advances and state-of-the-art technology in next-generation cooperative networks have paved the way for wireless energy cooperation between communicating nodes in which the idea is to power up the relay node through wireless energy harvesting $[14,15]$.

1.1. Related Works. The concept to simultaneously transmit both information and energy was first proposed by Varshney in [2], in which the fundamental trade-off for the capacityenergy function was characterized under the assumption of an ideal energy harvesting receiver. However, in practice, it is impossible to design an ideal energy harvesting receiver. Thus, [16] proposed two practical approaches based on power-splitting (PS) and time-switching (TS) mechanisms. Based on [16], Nasir et al. in [17] proposed two different relaying protocols, namely, PS-based relaying protocol (PSR) and TS-based relaying protocol (TSR). The performance of both TSR and PSR was studied for amplify-and-forward (AF) relay based one-way RF energy harvesting relay network, and it was concluded that, at a high transmission rate and low signal-to-noise-ratio (SNR) regions, the TSR protocol performs significantly better than the PSR protocol. Because of the distinct features of the two relaying protocols, the throughput analysis of decode-and-forward (DF) and AF relaying networks is different under energy harvesting constraints [18]. The throughput analysis of a PSR, TSR, and a combined time-power switching relaying (TPSR) [19] protocol for two-way AF relay network was studied in [20, 21], in which the results showed that, at high SNR and low transmission rate, TPSR protocol outperforms TSR and PSR. On the other hand, at high transmission rates and low SNR, the TSR protocol outperforms the TPSR and PSR protocols.

A two-way relay network based on the denoise-andforward relay with noncoherent differential binary phaseshift keying modulation has been studied in [22], while an energy harvesting and information processing network based on two-way multiplicative relay using PSR protocol was investigated in [23]. The numerical results showed that the proposed scheme outperforms the amplify-and-forward $\mathrm{EH}$ relaying technique. Ding et al. in [24] studied different power allocation strategies for power distribution in energy harvesting networks. In [25], Krikidis et al. studied the concept of energy transfer in a cooperative AF relay network based on wireless energy transfer, in which the performance of a greedy switching policy, where the relay node only transmits when its remaining power ensures decoding at the destination, was investigated. In addition, an optimal switching policy with global channel knowledge was also proposed, and it was shown that the greedy switching policy arises as an efficient solution. An outage probability analysis for energy harvesting multirelay networks is provided in [26]. The authors have studied two different relaying algorithms based on best relay selection and cooperative clustered-relying, and it has been shown that the former scheme outperforms the later in terms of outage probability. In [27], an energy harvesting-based weighed cooperative spectrum sensing scheme for cognitive radio networks is proposed. The authors have formulated a joint optimization problem to maximize the spectrum access probability of the secondary users by jointly optimizing the sensing time, a number of cooperative secondary users, and PS factor.

A geometric water-filling [28, 29] based on optimal power allocation scheme for cognitive radio (CR) multiple-inputmultiple-output (MIMO) systems with energy harvesting capabilities was proposed in [30]. The authors showed that the proposed algorithm has finite computations with a low degree of polynomial computational complexity. In [31], an extended version of a geometric water-filling approach proposed in [29] was used to maximize the throughput and minimize the transmission completion time of a hybrid energy source system. Their considered hybrid energy source system consists of two energy sources: (1) energy harvesting from the environment and (2) energy from the power grid. It has been shown that the optimal power allocation can be achieved by adopting the proposed sequence of water-filling algorithms. A similar geometric water-filling based resource scheduling scheme for the cellular network has been studied in [32].

Eirini Eleni Tsiropoulou et al. in [10] have introduced a wireless energy harvesting-based concept of joint consideration of interest-, physical-, and energy-related properties in the clustering and resource management processes of $\mathrm{M} 2 \mathrm{M}$ communication networks. In their proposed approach, the cluster-head supports its respective nodes to harvest and store energy in a stable manner via RF energy harvesting. The simulation results show that the proposed scheme significantly prolongs the operation of the overall M2M network. In [11], the authors have investigated the performance of ambient energy harvesting in a nonorthogonal multiple access (NOMA) based dual hope DF relay network. The relay nodes in the considered network are assumed to be energy constrained. With the help simulation results, the impact of relay selection under different successive interference cancellation (SIC) techniques is shown and it is concluded that the overall system performance is highly influenced by the efficiency of SIC techniques.

In [12], Vamvakas et al. have studied the issue of usercentric energy-efficient power management in an RF energy harvesting-based wireless sensor network. A low complexity, distributed, and adaptive energy transfer algorithm is proposed. The proposed algorithm aims to determine the optimal transmission power of power stations in the network. Their numerical results show that the proposed algorithm outperforms the existing state-of-the-art approaches in terms of network energy efficiency.

An interesting approach to extend the network lifetime via placing relay nodes at optimal locations is studied in [33]. The authors have addressed the problem of relay placement under the location constraint; i.e., the relay nodes can only be at set of candidate locations. The performance of an RF energy harvesting-based full duplex MIMO relay network is studied in [34]. With the help of numerical results it has been shown that the use of multiple antennas at both source and destination nodes can significantly improve the overall system performance. A joint optimization of positioning and 


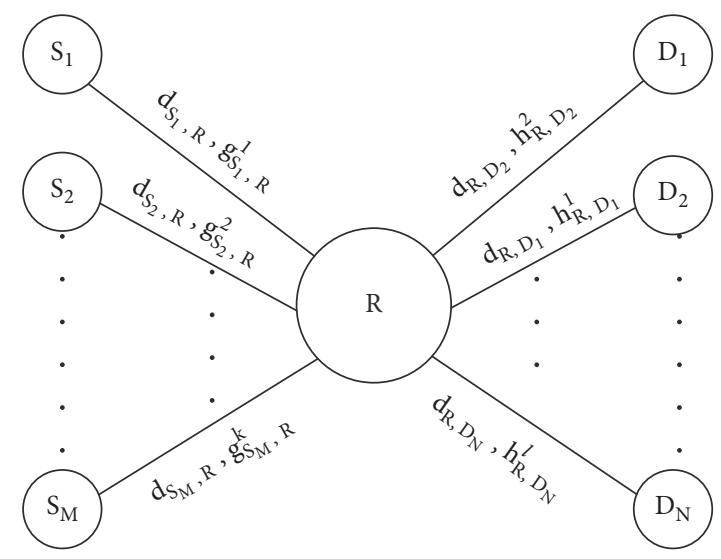

FIGURE 1: System model of energy harvesting-based multichannel relay network.

routing for designing wireless body area sensor networks with traffic uncertainty is developed in [35].

1.2. Objective and Contribution. The idea of relays for coverage extension in wireless sensor networks has been well established and widely accepted [36]. In cooperative wireless networks, the battery power of the cooperating nodes (such as relay nodes) is usually limited, and to actively perform their role in the network, these nodes may need to rely on an additional charging mechanism $[11,12]$. In this paper, we propose a dynamic wireless energy harvesting and optimal power distribution scheme for a multipoint-to-multipoint energy harvesting-based DF relay network. The main contributions of the present paper are summarized as follows:

(i) Unlike most of the works discussed in the previous section where, in a typical point-to-point relaying network, two source nodes exchange data via a relay node, this paper considers a network where multiple source nodes communicate with their respective destination nodes via intermediate energy-constrained DF relay.

(ii) Since the relay is an energy-constrained node and has no energy of its own, it harvests energy from multiple received source signals and then distributes the harvested energy among all possible destination nodes. For energy harvesting at the relay node, we adopt PSR protocol [17].

(iii) For an in-depth performance analysis of the proposed system model, two different transmission modes, namely, delay tolerant mode and delay nontolerant mode, are considered. For both of these transmission modes, we have proposed dynamic energy harvesting and optimal power distribution schemes. More specifically, in delay tolerant transmission mode, optimal energy is harvested and distributed by relay node for each individual source-to-destination link. In delay nontolerant transmission mode, the relay node dynamically harvests the energy from all source signals power based on their received signal-to-noiseratio. Then this harvested energy is distributed among all possible destination nodes using the geometric water-filling technique [28]. More details about both of these transmission modes are provided in Section 4 .

(iv) Furthermore, unlike most of the previous studies [3$18,37,38]$, this paper considers a more realistic and practical approach of nonlinear energy efficiency for energy harvesting at relay [39].

(v) With the help of numerical results, useful and detailed practical insights of our proposed scheme are provided.

1.3. Organization. The remainder of the paper is organized as follows. The considered system model is presented in Section 2. The generalized procedures for information processing and energy harvesting using PSR protocol are explained in Section 3. The details on our proposed optimal power distribution and energy harvesting scheme for both delay tolerant and delay nontolerant transmission modes are provided in Sections 4. Section 5 provides the detailed discussion of numerical results. Finally, the paper is concluded in Section 6.

\section{System Model}

We consider an RF energy harvesting-based multichannel multipair DF relay network as shown in Figure 1. In the proposed network, we define $\mathbb{S}=\left\{S_{m} \mid S_{m}=1,2, \cdots, M\right\}$ and $\mathbb{D}=\left\{D_{n} \mid D_{n}=1,2, \cdots, N\right\}$ as the sets of source and destination nodes in the network with cardinality $M$ and $N$, respectively. In our proposed scheme, information is transmitted from source node $S_{m}$ (where $S_{m} \in \mathbb{S}$ ) to its respective destination node $D_{n}\left(D_{n} \in \mathbb{D}\right)$, via an intermediate energyconstrained DF relay node $\mathrm{R}$ using orthogonal channels. It is assumed that there is no direct link between the source and destination nodes, and the respective SNRs of the channels between the communicating nodes are less than the minimum required threshold SNR for effective communication. Therefore, to assist the information transmission between communicating nodes, an intermediate relay node $(\mathrm{R})$ is used [40]. 
TABLE 1: Table of notations.

\begin{tabular}{|c|c|c|}
\hline & Notation & Explanation \\
\hline 1 & $M$ & Total number of source nodes and source-to-relay channels \\
\hline 2 & $N$ & Total number of destination nodes and relay-to-destination channels \\
\hline 3 & $S_{m}$ & Source node $m$, where $m=1 \cdots M$ \\
\hline 4 & $D_{n}$ & Destination node $n$, where $n=1 \cdots N$ \\
\hline 5 & $\mathrm{R}$ & Relay Node \\
\hline 6 & $d_{S m, R}$ & Distance between source node $S_{m}$ and relay node R \\
\hline 7 & $d_{R, D_{n}}$ & Distance between relay node $\mathrm{R}$ and destination node $D_{n}$ \\
\hline 8 & $\rho_{m}^{* k}$ & Dynamically adjusted power splitting factor for signal received from node $m$ via channel $k$, where $k=1,2, \cdots, M$ \\
\hline 9 & $P_{S_{m}, R}^{k}$ & Power of source node $S_{m}$ to relay node R via channel $k$, where $k=1,2, \cdots, M$ \\
\hline 10 & $P_{R, D_{n}}^{l}$ & Power allocated by relay node $\mathrm{R}$ to destination node $D_{n}$ on channel $l$, where $l=1,2, \cdots, N$ \\
\hline 11 & $g_{S_{m}, R}^{k}$ & Channel gain between source node $S_{m}$ and relay node R via channel $k$, where $k=1,2, \cdots, M$ \\
\hline 12 & $h_{R, D_{n}}^{l}$ & Channel gain between relay node $\mathrm{R}$ and destination node $D_{n}$ on channel $l$, where $l=1,2, \cdots, N$ \\
\hline 13 & $n_{R}^{a}, n_{R}^{c}$ & Antenna and conversion nose at relay, respectively \\
\hline 14 & $n_{D}^{a}, n_{D}^{c}$ & Antenna and conversion noise at destination, respectively \\
\hline 15 & $\sigma_{R}^{2}$ & Combined variance of both antenna and conversion noise at relay $\mathrm{R}$ \\
\hline 16 & $\sigma_{D}^{2}$ & Combined variance of both antenna and conversion noise at destination node $\mathrm{D}$ \\
\hline 17 & $\widehat{P}$ & Remaining power at relay \\
\hline 18 & $\widetilde{P}$ & Total power harvested at relay from all source signals \\
\hline 19 & $l^{*}$ & Highest step which is under water \\
\hline 20 & $\Gamma_{l}$ & Step depth of stair $l$ where $\Gamma_{l}=d_{R, D_{n}}^{\alpha} \sigma_{D}^{2} /\left|h_{R, D_{n}}^{l}\right|^{2}$ \\
\hline 21 & $U_{D_{n}}$ & Achievable throughput at destination node $D_{n}$ \\
\hline 22 & $\eta$ & Energy conversion efficiency \\
\hline
\end{tabular}

In Figure $1, d_{S_{m}, R}$ and $g_{S_{m}, R}^{k}$ represent the distance and channel coefficient between source node $m$ and relay node $\mathrm{R}$ via frequency channel $k$ (where $k=1,2, \cdots, M$ ), respectively. Similarly, $d_{R, D_{n}}$ and $h_{R, D_{n}}^{l}$ represent the distance and channel coefficient between relay node $\mathrm{R}$ and destination node $n$ via channel $l$ (where $l=1,2, \cdots, N$ ), respectively. Note that, since each node is using a single orthogonal frequency channel for communication, the total numbers of source-torelay and relay-to-destination channels are equal to the total number of source nodes and destination nodes, respectively. The orthogonal channels are considered in order to avoid cochannel interference. The channel coefficients are assumed to be quasi-static block-fading, which means that the channel state is constant and does not vary over a transmission block time $T$. It is also assumed that they are independent and identically distributed in each time block following a Rayleigh distribution. The path-loss model considered in this paper is a distance-dependent path-loss model $d^{-\alpha}$, where $\alpha$ is the path-loss exponent and $d$ is the propagation distance. The use of such path-loss and channel model is motivated by the previous work done in this research area [7, 17, 18, 37, 38, 41]. For the sake of readers' convenience, all the notations used in this paper are summarized in Table 1.

Furthermore, we assume that the relay node $\mathrm{R}$ is an energy-constrained node. Therefore, it first harvests energy from the received source signals and then utilizes this harvested energy to forward these received signals to their respective destinations.

\section{Energy Harvesting and Information Processing in PS-Based DF Relay}

In this section, a generalized energy harvesting and information processing procedure for a PS-based energy-constrained DF relay network is provided. In order to harvest energy at relay node $\mathrm{R}$, we consider the PS-based relaying (PSR) protocol proposed in $[16,17]$. According to PSR protocol, the entire received power of the source signal is split into two portions by using a power splitter. One portion of power is used for energy harvesting while the other is used for information processing. Figures 2(a) and 2(b) depict the transmission time-block structure and block diagram, respectively, for PSR protocol. Please note that $P_{S_{m}, R}^{k}$ denotes the power of the signal received from source node $S_{m}$ via channel $k$ and $\mathrm{T}$ stands for the total transmission time which is divided into two halves; the first T/2 half is used for source-to-relay transmission, and the second $\mathrm{T} / 2$ half is used for relay-to-destination transmission. According to PSR protocol, during the first half a certain fraction of the received signal power $\rho_{m} P_{S_{m}, R}^{k}$ is used for energy harvesting and the remaining power $\left(1-\rho_{m}\right) P_{S_{m}, R}^{k}$ is used for information processing, where $\rho_{m}$ denotes the power-splitting factor of 


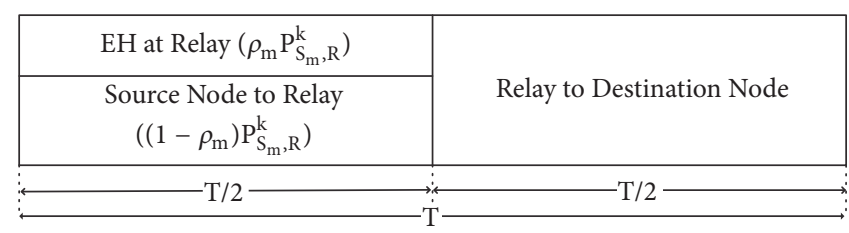

(a)

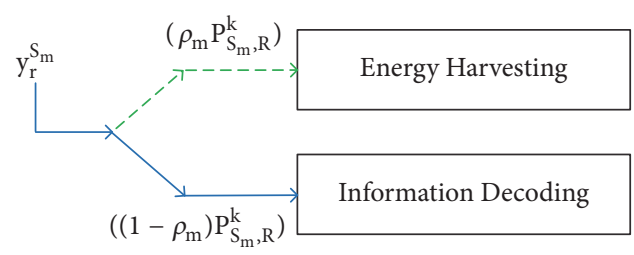

(b)

FIGURE 2: (a) Transmission time-block structure for PSR protocol; (b) block diagram of PSR based relay receiver.

signal received from source node $m$. The value of $\rho$ ranges between 0 and 1 (i.e., $0 \leq \rho \leq 1$ ).

The signal received at relay R from source node $S_{m}$ can be written as

$$
y_{r}^{S_{m}}=\sqrt{\frac{P_{S_{m}, R}^{k}}{d_{S_{m}, R}^{\alpha}}} g_{S_{m}, R}^{k} x_{S_{m}}+n_{R}^{a},
$$

where $x_{S_{m}}$ is the signal information received from source node $S_{m}$. The additive white Gaussian noise (AWGN) at the receiving antenna of the relay node is denoted by $n_{R}^{a}$. A portion of the received signal $y_{r}^{S_{m}}$ is sent to the energy harvesting circuit. In case of linear energy efficiency model [17], the harvested energy and power can, respectively, be expressed as

$$
\begin{aligned}
E H_{S_{m}}^{L} & =\frac{\eta \rho_{m} P_{S_{m}, R}^{k}\left|g_{S_{m}, R}^{k}\right|^{2} T}{2 d_{S_{m}, R}^{\alpha}}, \\
\text { and } P_{S_{m}}^{L} & =\frac{E H_{S_{m}}^{L}}{T / 2},
\end{aligned}
$$

where $\eta$ is the efficiency of the energy harvesting receiver and its value ranges between 0 and 1 (i.e., $0 \leq \eta \leq 1$ ). Thus, the total harvested energy at relay node from $M$ source signals can be expressed as

$$
E H_{T}^{L}=\eta \frac{T}{2} \sum_{k=m=1}^{M} \frac{\rho_{m} P_{m, r}^{k}\left|g_{S_{m}, R}^{k}\right|^{2}}{d_{S_{m}, R}^{\alpha}} .
$$

The above equation can also be written in terms of total harvested power as

$$
\widetilde{P}^{L}=\frac{E H_{T}^{L}}{T / 2}=\eta \sum_{k=m=1}^{M} \frac{\rho_{m} P_{S_{m}, R}^{k}\left|g_{S_{m}, R}^{k}\right|^{2}}{d_{S_{m}, R}^{\alpha}} .
$$

However, in practice the energy conversion efficiency exhibits nonlinear behavior [42]. Therefore, for more practical analysis of our considered network model, we have also adopted a nonlinear energy conversion efficiency model provided in [39]. Based on this nonlinear energy conversion efficiency model, the power harvested from a source signal at relay node can be expressed as
$P_{S_{m}}^{N L}=\frac{\left[\Xi_{S_{m}}-\lambda_{S_{m}} \Omega_{S_{m}}\right]}{1-\Omega_{S_{m}}}$,

where $\Xi_{S_{m}}$

$$
\begin{aligned}
& \quad=\frac{\lambda_{S_{m}}}{1+\exp \left(-x_{S_{m}}\left(\rho P_{S_{m}, R}^{k}\left|g_{S_{m}, R}^{k}\right|^{2} / d_{S_{m}, R}^{\alpha}-y_{S_{m}}\right)\right)}, \\
& \text { and } \Omega_{S_{m}}=\frac{1}{1+\exp \left(x_{S_{m}} y_{S_{m}}\right)} .
\end{aligned}
$$

The $\Xi_{S_{m}}$ and $\Omega_{S_{m}}$ are the traditional logistic functions with respect to the received RF power and constant to ensure a zero-input/zero-output response for energy harvesting, respectively. $\lambda_{S_{w}}$ is a constant which denotes the maximum power harvested at relay node when the energy harvesting circuit is saturated. Likewise, $x_{S_{m}}$ and $y_{S_{m}}$ are constants related to the circuit specifications such as the diode turnon voltage, capacitance, and resistance [39]. Finally, in case of nonlinear energy harvesting, the total amount of energy harvested at relay node can be written as

$$
\widetilde{P}^{N L}=\sum_{k=m=1}^{M} P_{S_{m}}^{N L} .
$$

After successful energy harvesting and passband-tobaseband conversion, the received signal (from source node $S_{m}$ ) at the information processing unit of relay $\mathrm{R}$ is given by

$$
\tilde{y}_{r}^{S_{m}}=\sqrt{\frac{\left(1-\rho_{m}\right) P_{S_{m}, R}^{k}}{d_{S_{m}, R}^{\alpha}}} g_{S_{m}, R}^{k} x_{S_{m}}+\sqrt{1-\rho_{m}} n_{R}^{a}+n_{R}^{c},
$$

where $n_{R}^{c}$ denotes the AWGN due to RF-to-baseband conversion. The received SNR (from source node $S_{m}$ via channel $k$ ) at relay node $\mathrm{R}$ can be expressed as

$$
\gamma_{r}^{S_{m}^{k}}=\frac{\left(1-\rho_{m}\right) P_{S_{m}, R}^{k}\left|g_{S_{m}, R}^{k}\right|^{2}}{d_{S_{m}, R}^{\alpha} \sigma_{R}^{2}},
$$

where $\sigma_{R}^{2}$ is the combined variance of AWGN $N_{R}$ at the relay node (where $N_{R} \triangleq \sqrt{1-\rho_{m}} n_{R}^{a}+n_{R}^{c}$ ).

The DF relay decodes the received signal $\tilde{y}_{r}^{S_{m}}$ and forwards it to its respective destination $D_{n}$ with transmit power 
$P_{R, D_{n}}^{l}$. The received signal at destination node $D_{n}$ can be expressed as

$$
y_{r}^{D_{n}}=\sqrt{\frac{P_{R, D_{n}}^{l}}{d_{R, D_{n}}^{\alpha}}} h_{R, D_{n}}^{l} x_{S_{m}}+n_{D}^{a}+n_{D}^{c},
$$

where $n_{D}^{a}$ and $n_{D}^{c}$ are the antenna and conversion AWGNs at the destination node, respectively. The received SNR at destination node $D_{n}$ (from relay node $\mathrm{R}$ via channel $l$ ) can be expressed as

$$
\gamma_{d}^{D_{n}^{l}}=\frac{P_{R, D_{n}}^{l}\left|h_{R, D_{n}}^{l}\right|^{2}}{d_{R, D_{n}}^{\alpha} \sigma_{D}^{2}}
$$

where $\sigma_{D}^{2}$ is the combined variance of AWGN $N_{D}$ at the destination node (where $N_{D} \triangleq n_{D}^{a}+n_{D}^{c}$ ). In case of delay tolerant transmission mode, the achievable throughput at destination nodes can be estimated as

$$
\begin{aligned}
& U_{D_{n}}^{D T}=\frac{1}{2} \log \left(1+\min \left(\gamma_{r}^{S_{m}^{k}}, \gamma_{d}^{D_{n}^{l}}\right)\right)=\frac{1}{2} \log (1 \\
& \left.\quad+\min \left(\frac{\left(1-\rho_{m}\right) P_{S_{m}, R}^{k}\left|g_{S_{m}, R}^{k}\right|^{2}}{d_{S_{m}, R}^{\alpha} \sigma_{R}^{2}}, \frac{\eta \rho_{m} P_{S_{m}, R}^{k}\left|g_{S_{m}, R}^{k}\right|^{2}\left|h_{R, D_{n}}^{l}\right|^{2}}{d_{S_{m}, R}^{\alpha} d_{R, D_{n}}^{\alpha} \sigma_{D}^{2}}\right)\right) .
\end{aligned}
$$

On the other hand, for delay nontolerant case the achievable throughput at destination nodes is determined based on the fact that the source signal received at destination is successfully decoded or not. In other words, for successful decoding, the received SNR of source signal at relay should be greater than a predefined threshold SNR. This can also be defined in terms of outage probability at node $\mathrm{R}$ which can be expressed as

$$
\begin{aligned}
P_{\text {out }, R}^{S_{m}^{k}} & =\gamma_{r}^{S_{m}^{k}}<\gamma \\
& =\frac{\left(1-\rho_{m}\right) P_{S_{m}, R}^{k}\left|g_{S_{m}, R}^{k}\right|^{2}}{d_{S_{m}, R}^{\alpha} \sigma_{R}^{2}}<\gamma,
\end{aligned}
$$

where $\gamma=2^{B}-1$ is the minimum threshold SNR for successful decoding and $B$ is the source node transmission rate. Based on (11) and (15), the achievable throughput at destination node for delay nontolerant transmission mode can be estimated as

$$
U_{D_{n}}^{D N T}=\left(1-P_{\text {out }, R}^{S_{m}^{k}}\right) \frac{B}{2} .
$$

Consequently, for both transmission modes the sum-rate, which is the sum of all achievable data rates at destination nodes, can be expressed as

$$
\text { sum }- \text { rate }=\sum_{n=1}^{N} U_{D_{n}}^{D T / D N T} .
$$

It can be observed from (11), (13), (14), and (17) that the achievable rate at destination nodes is highly dependent on the transmission power of relay $P_{R, D_{n}}^{l}$, which further depends on the power-splitting factor $\rho_{m}$ at the relay. In this paper, we characterize the performance of our considered system by its achievable sum-rate. Therefore, the achievable sumrate at the destination is used as a performance metric. In the next section, we will develop an efficient power distribution and energy harvesting scheme for both transmission modes which aims to maximize the achievable sum-rate at the destination.

\section{Proposed Optimal Power Distribution and Energy Harvesting}

In this section, we focus on the design of an efficient transmit power distribution and dynamic energy harvesting mechanism for both delay nontolerant and delay tolerant transmission modes.

4.1. Delay Nontolerant Transmission Mode. In delay nontolerant transmission mode, the achievable rate at destination highly depends on the amount of energy harvested at relay and transmit power of relay. Therefore an efficient energy harvesting and transmit power allocation scheme will result in improved achievable sum-rate at destination. Consequently, for delay nontolerant transmission mode the generalized optimization problem for sum - rate maximization can be formulated as

$$
\begin{aligned}
\max _{\left\{P_{R, D_{n}}^{l}\right\}_{n=1}^{N}} & \sum_{n=1}^{N} U_{D_{n}}^{D N T} \\
\text { s.t. } & 0 \leq P_{R, D_{n}}^{l} \leq \widetilde{P} \\
& \sum_{n=1}^{N} P_{R, D_{n}}^{l}=\widetilde{P},
\end{aligned}
$$

where $\widetilde{P}$ (for notational simplicity, $\widetilde{P}$ is used instead of $\widetilde{P}^{L}$ and $\widetilde{P}^{N L}$ ) is the total transmit power available at the relay node. In other words, $\widetilde{P}$ denotes the total harvested power from all source nodes. The first constraint in (19) ensures the limitation on maximum power allocation by the relay. Likewise, the second constraint means that the total transmit power consumed by relay should be equal to the total harvested power. In other words, for each transmission block time, the relay should consume all the harvested power.

Our proposed scheme for delay nontolerant transmission mode has two main phases: dynamic energy harvesting and optimal transmit power distribution. The relay first harvests the energy from all received signals and then distributes it among all possible relay-to-destination channels using the geometric water-filling technique. The water-filling distributes the power in such a manner that the sum of data rates at all destination nodes is maximized. The decision on how much energy should be harvested from each received signal (value of $\rho$ ) is made considering two major factors: (1) the power of the received signal at the relay and (2) the results of water-filling based power distribution. The detailed procedure and discussion on both phases are provided in next subsections. 


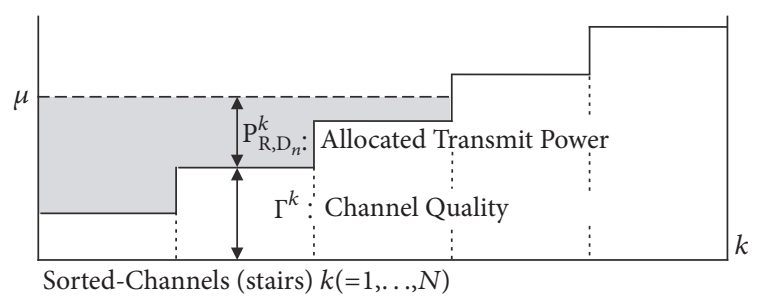

FIGURE 3: Illustration of geometric water-filling.

\subsubsection{Geometric Water-Filling Based Optimal Transmit Power} Distribution. In transmit power distribution phase, the relay node distributes the available harvested power using geometric water-filling approach [28]. In order to maximize the sum of data rates of all channels, water-filling approach allocates more power to the channels with higher gains [43]. Figure 3 shows an example of a water-filling based transmit power allocation to $N$ different relay-to-destination channels. In order to maximize the sum of data rates of overall system $\sum_{n=1}^{N} U_{D_{n}}$, water-filling algorithm aims to determine the transmission power of relay node $\mathrm{R}$ for each destination node $D_{n}$ via its orthogonal channel $l$.

In order to map our proposed scheme into water-filling paradigm, we consider $l$ as the index of the stair (where $l=1,2, \cdots, N)$. Here we assume that stairs are arranged in descending order; in other words, the channels with higher gains are prioritized. The transmit power assigned by the relay to a destination node $D_{n}$ at channel $l$ is the level of water poured into stair $l$ and is represented as $P_{R, D_{n}}^{l}$. Likewise, $\mu$ is the finalized water level of the water tank (i.e., total available power at relay) and $\Gamma^{l}$ represents the step depth of stair $l$ which can be calculated as

$$
\Gamma^{l}=\frac{d_{R, D_{n}}^{\alpha} \sigma_{D}^{2}}{\left|h_{R, D_{n}}^{l}\right|^{2}} .
$$

The conventional way to solve the water-filling problem is to solve a nonlinear system from the KKT conditions and then find the water level $(\mu)[44]$. It has been shown in $[28,29]$ that a geometric water-filling approach incurs less complexity and it can compute the exact solution as of conventional method without solving any nonlinear system to determine water level(s). Unlike conventional method, where we try to compute the water level $\mu$, which is a nonnegative real number, the geometric technique aims to determine the highest step under water which is denoted by $l^{*}$ and is an integer ranging from 1 to $N$. To further elaborate the concept of $l^{*}$, we provide an example case in Figure 4(a). It can be observed from Figure 4(a) that the highest step under water is step 3 , and therefore, in this case, $l^{*}=3$. The highlighted area shows the power allocated to step 3 , and it is denoted by $P_{R, D_{n}}^{3^{*}}$.

In order to find the highest step under water $l^{*}$ without any knowledge of the water level $\mu$, we first compute the $R(l)$ which can be defined as the volume of water above step $l . R(l)$ can be calculated as

$$
\begin{aligned}
R(l) & =\left\{\widetilde{P}-\left[\sum_{j=1}^{l-1}\left(\frac{d_{R, D_{n}}^{\alpha} \sigma_{D}^{2}}{\left|h_{R, D_{n}}^{l}\right|^{2}}-\frac{d_{R, D_{n}}^{\alpha} \sigma_{D}^{2}}{\left|h_{R, D_{n}}^{j}\right|^{2}}\right)\right]\right\}^{+}, \\
& =\left\{\widetilde{P}-\left[\sum_{j=1}^{l-1}\left(\Gamma^{l}-\Gamma^{j}\right)\right]\right\}^{+},
\end{aligned}
$$

$$
\text { for } l=1,2, \cdots, N \text {, }
$$

where $\{\cdot\}^{+}$indicates that the volume of water above step $l$ cannot be a negative value. The shaded area in Figure 4(b) shows the concept of water volume above step $l(R(l))$, when $l=2$. Based on (21), the highest step under water $l^{*}$ (e.g., step 3 in Figure 4(b)) can be computed as

$$
\begin{aligned}
& l^{*}=\max \left(l \mid\left\{\tilde{P}-\left[\sum_{j=1}^{l-1}\left(\Gamma^{l}-\Gamma^{j}\right)\right]\right\}^{+}>0,1 \leq l\right. \\
& \quad \leq N) \\
& =\max (l \mid R(l)>0,1 \leq l \leq N) .
\end{aligned}
$$

According to geometric Water-filling [28], the explicit optimal solution of (19) based on (21) and (22) can be found as

$$
\begin{aligned}
& P_{R, D_{n}}^{l} \\
& \quad=\left\{P_{R, D_{n}}^{l^{*}}+\left(\Gamma^{l^{*}}-\Gamma^{l}\right), \text { for } 1 \leq l \leq l^{*} 0,\right. \text { otherwise, }
\end{aligned}
$$

where the power level of $l^{*}$ can be determined as

$$
P_{R, D_{n}}^{l^{*}}=\frac{R\left(l^{*}\right)}{l^{*}} .
$$

Note that no power has been allocated to the channels whose index is higher than $l^{*}$. On the other hand, power levels for channels whose index is lower than $l^{*}$ are determined according to (23), where $P_{R, D_{n}}^{l^{*}}$ is added with the difference of step depth of corresponding step and $l^{*}$ th step.

The pseudocode of our proposed geometric water-filling based power distribution scheme is provided in Phase-II of Algorithm 1. The algorithm begins with channel sorting and finding the highest step underwater $l^{*}$ (see (22)). In other words, the relay first determines the maximum number of destination nodes that can be accommodated in a manner where the overall system throughput is maximized. Then the power allocated to each channel whose index is below $n^{\prime *}$ is calculated according to (23).

4.1.2. Dynamic Energy Harvesting Scheme. It is obvious that the value of power-splitting factor $\rho$ plays a vital role in PS-based wireless energy harvesting relay networks. In our proposed energy harvesting scheme for delay nontolerant transmission scheme, the value of $\rho$ for each received signal at relay node R is decided based on two factors: (1) power level of the received signal and (2) the availability of transmitting 


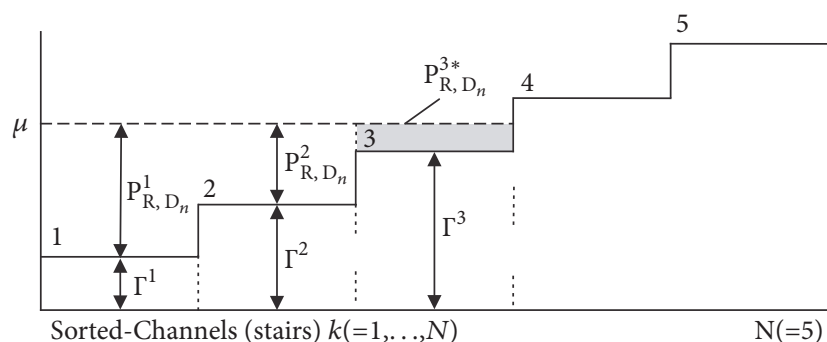

(a) Illustration of the highest step under water $\left(l^{*}=3\right)$

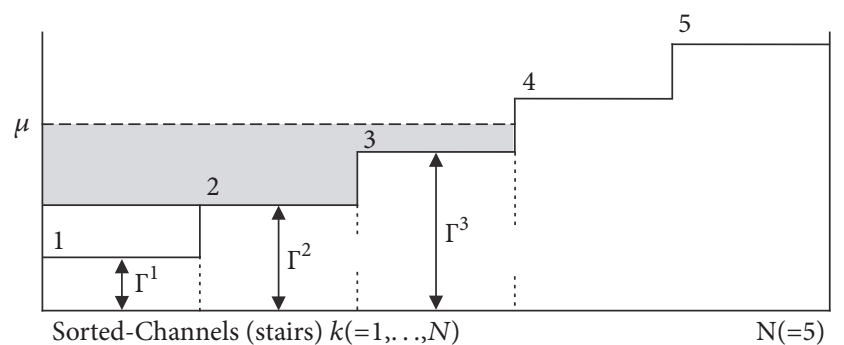

(b) Illustration of the water volume above step $l R(l)$ (where, $l=2$ )

FIgURE 4: An illustration example to explain the highest step under water $l^{*}$ and water volume above step $l R(l)$.

(1) Initialize $Y ; Z \widetilde{P} / /$ where $Y$ is total number of successfully decoded source-to-relay channels and $Z$ is total number of relay-todestination channels to whom power has been allocated by the relay.

(2) while $Y \neq Z$ do

(3) procedure Energy Harvesting (Phase-I)

(4) for all received signals $\left\{y_{r}^{S_{m}}\right\}_{m=1}^{M}$ do

(5) if $\gamma_{r}^{S_{m}^{k}} \geq \gamma$ and the sources' corresponding $k$ channel is flaggednegative then

(6) Set the value of power splitting factor $(\rho)$ according to (26) \& (27) and harvest the energy according to ((2) or (6)).

(7) $\quad$ Set $Y \longleftarrow Y+1$

(8) else

(9) Set the value of $\rho=1$ (see eq. (27)) and harvest energy according to (2).

(10) end if

(11) Update $\widetilde{P}$

(12) end for

(13) end procedure

(14) procedure Power Distribution (Phase-II)

(15) Set $\widetilde{P}=\widehat{P}$

(16) Exclude the destinations/channels whose source received SNR is less than $\gamma$ and sort the remaining destinations/ channels in descending order w.r.t to their channel gains.

(17) Find the highest step underwater $l^{*}$ according to (22).

(18) for all relay-to-destination channels (i.e. $l=1,2, \cdots, N)$ do

(19) $\quad$ if $l \leq l^{*}$ then

(20) Mark it as flagged negative

(21) else

(22) Mark it as flagged positive

(23) $\quad$ set $Z=l^{*}$

(24) end if

(25) end for

(26) Distribute $\widehat{P}$ by allocating power to all flagged positive channels according to ((23) \& (24))

(27) end procedure

(28) end while

Algorithm 1: Energy harvesting and transmit power distribution.

power for that particular signal, in order to forward it to its corresponding destination. If the power of any received signal at the relay node $\widetilde{\gamma}$ is less than the decoding threshold (i.e., $\gamma_{r}^{S_{m}^{k}}<\gamma$, where $\gamma$ is the decoding threshold SNR), the whole received signal is harvested $(\rho=1)$. It is because the received signal cannot be correctly decoded at the relay.

As mentioned earlier, during water-filling based power distribution phase some relay-to-destination channels (i.e., $l>l^{*}$ ) might not receive any power (see (23)). Therefore, the second factor can be divided into two cases.
Case 1. During the geometric WF based power distribution phase, if there is no power available at relay for the successful transmission of a particular signal to its corresponding destination (see lines (9) and (22) of Algorithm 1), the whole received signal is harvested $(\rho=1)$.

Case 2. On the other hand, if the relay has enough power available for the successful transmission of a particular signal to its corresponding destination (see lines (19)-(20) of Algorithm 1), it uses the decoding threshold power for signal 
processing and harvests the remaining signal power (see lines (5)-(6) of Algorithm 1).

In summary, for successful decoding of received signal at relay node $\mathrm{R}$ the following condition should be satisfied:

$$
\begin{aligned}
\gamma_{r}^{S_{m}^{k}} & =\frac{\left(1-\rho_{m}\right) P_{S_{m}, R}^{k}\left|g_{S_{m}, R}^{k}\right|^{2}}{d_{S_{m}, R}^{\alpha} \sigma_{R}^{2}} \geq \gamma, \text { it means } \\
\rho_{m}^{k} & =1-\frac{d_{S_{m}, R}^{\alpha} \gamma \sigma_{R}^{2}}{P_{S_{m}, R}^{k}\left|g_{S_{m}, R}^{k}\right|^{2}} .
\end{aligned}
$$

Thus, the ideal power-splitting factor $\rho^{*}$ for a signal (received from source node $m$ via channel $k$ ) can be decided according to

$$
\begin{aligned}
\rho_{m}^{* k} & =\left\{\max \left(0,1-\frac{d_{S_{m}, R}^{\alpha} \gamma}{P_{S_{m}, R}^{k}\left|g_{S_{m}, R}^{k}\right|^{2}}\right), \text { if } P_{R, D_{n}}^{l}\right. \\
& >0 \text { and } \gamma_{r}^{S_{m}^{k}} \geq \gamma 1, \text { otherwise. }
\end{aligned}
$$

For a DF relay network operating in delay nontolerant transmission mode, the power-splitting strategy provided in (27) is ideal. More specifically, if the value of powersplitting factor $\rho$ is greater than $\rho^{*}$, small portion of signal power is used for energy harvesting, and unnecessarily extra power is allocated for signal decoding (whereas relay node only requires $\left(1-\rho_{m}^{* k}\right) P_{S_{m}, R}^{k}\left|g_{S_{m}, R}^{k}\right|^{2} / d_{S_{m}, R}^{\alpha} \sigma_{R}^{2}$ for guaranteed decoding) which results in waste of valuable power resource. On the other hand, if the value of power-splitting factor $\rho$ is less than $\rho^{*}$, relay node utilizes more power for energy harvesting which leads to the decoding failure of the source signal.

The pseudocode of our proposed energy harvesting mechanism is given in Phase-I of Algorithm 1. The algorithm begins with finding the power level of a received signal at relay node $\mathrm{R}$. Then it checks the available power at $\mathrm{R}$ for this particular signal in order to forward it to its respective destination. Based on these findings, the relay obtains the value of power-splitting factor $\rho^{*}$ according to (27).

4.2. Delay Tolerant Transmission Mode. In case of delay tolerant transmission mode, the power allocation is performed in a noncooperative manner. More specifically, the amount of energy harvested from a particular source signal is solely allocated to its corresponding destination. Such a noncooperative strategy is well suited for delay tolerant transmission mode because the relay node can decode the received signal unconditionally. In other words, for the successful decoding of received signal, the relay in delay tolerant mode does not necessarily require a minimum threshold SNR. Once a portion of received signal (after energy harvesting) is successfully decoded, the relay then utilizes the power harvested from the same signal to forward it to its corresponding destination. In such a noncooperative scenario the powersplitting factor $(\rho)$ plays a vital role to maximize the end-toend achievable throughput. For delay tolerant transmission mode, the generalized optimization problem to maximize the achievable throughput of an individual link can be formulated as

$$
\begin{array}{ll}
\max _{\rho_{m}} & U_{D_{n}} \\
\text { s.t. } & 0 \leq \rho_{m} \leq 1
\end{array}
$$

where the bound in (28) ensures that the value of $\rho$ should not exceed its $(0,1)$ limits. Since the above optimization problem is a concave function of $\rho$ and can be optimized using any standard numerical optimization method [45], for our case, we have used interior point method.

\section{Performance Evaluation}

This section numerically evaluates the performance of our proposed multipoint-to-multipoint DF relay network. The performance of two different transmission modes, delay tolerant and delay nontolerant transmission, are evaluated under the consideration of linear and nonlinear energy harvesting models. The values of different simulation parameters are source nodes transmit power $P_{S}=1$ Joules $/ \mathrm{sec}$; distances from source to relay $\left(d_{S_{m}, R}\right)$ and from relay to destination $\left(d_{R, D_{n}}\right)$ are normalized to unit value. To reflect the environment of an urban cellular network, the value of path-loss exponent $\alpha$ is set to 2.7 [46]. It is assumed that the noise factors at all nodes are equal and the values of combined noise variances on both relay and destination nodes are set to $\sigma_{R}^{2}=\sigma_{D}^{2}=0.02$. For the nonlinear energy harvesting model, the values of $\lambda_{S_{m}}, x_{S_{m}}$, and $y_{S_{m}}$ are set to $20 \mathrm{~mW}$, 6400 , and 0.003 . These values are adopted using curve fitting and measurement date from $[39,42]$. The results are obtained by averaging a large number of channel realizations.

For both delay tolerant and delay nontolerant transmission models, the effect of source transmit power $P_{S}$ on achievable sum-rate is depicted in Figures 5 and 6, respectively. It can be observed that the network sum-rate in linear energy harvesting model significantly increases as the transmit power of source nodes increases. This is because, at higher source transmit power, more energy is harvested at the relay (see (2)), which results in increased throughput power at the destination. However, in practice, the energy conversion efficiency exhibits a nonlinear behavior [39]. Therefore, it can be observed from Figures 5 and 6 that, in case of nonlinear energy harvesting model, the network sum-rate does not significantly change after a certain value of $P_{s}$. It is due to the fact that the energy harvesting circuit at relay follows a nonlinear behavior (see (6)), and after a certain point, it cannot harvest any more energy even if the input power is increased.

The achievable sum-rate with varying numbers of source/destination pairs (SDPs) of both delay tolerant and delay nontolerant transmission modes is shown in Figures 7 and 8, respectively. It is shown that in both transmission modes the network sum-rate linearly increases as the number of source/destination pairs in the network increases. However, the delay tolerant mode achieves significantly higher sum-rate than delay nontolerant mode. Furthermore, it can 


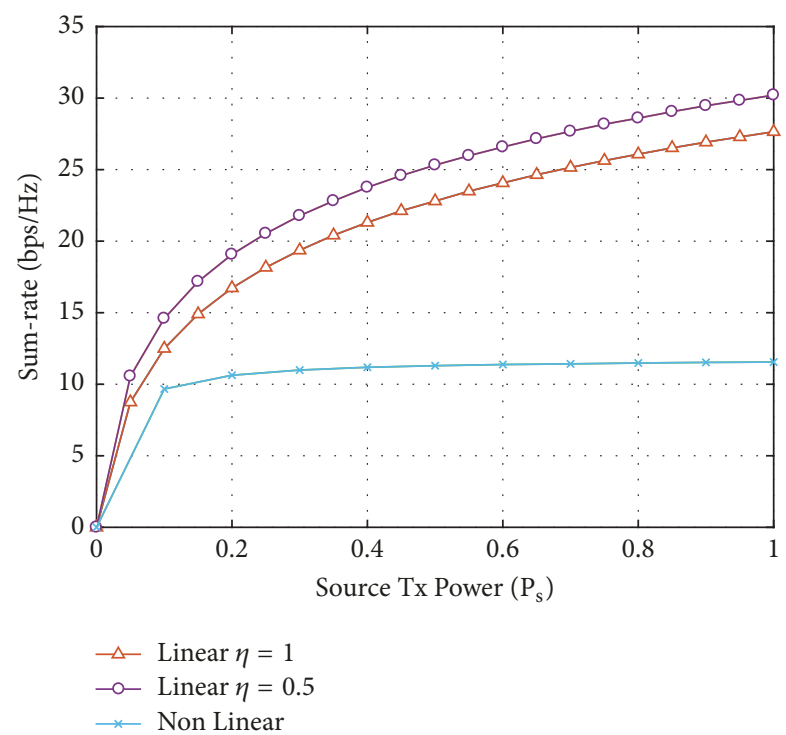

FigURE 5: Network sum-rate of proposed network in delay tolerant transmission mode with varying values of source transmission power $\left(P_{S}\right)$, where no. of $S D P s=10, d_{S_{m}, R}=d_{R, D_{n}}=1$, and $\sigma_{R}^{2}=\sigma_{D}^{2}=0.02$.

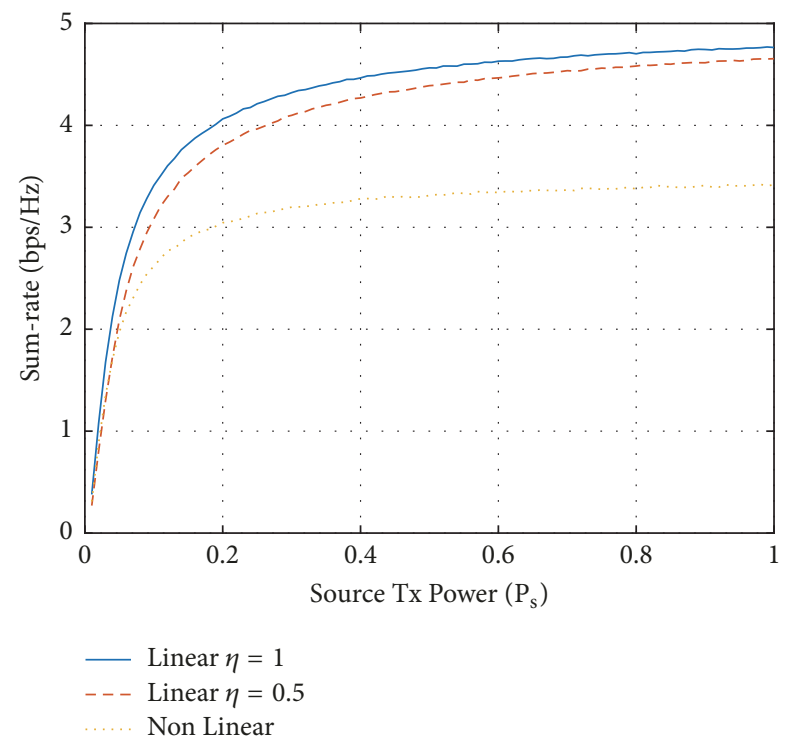

Figure 6: Network sum-rate of proposed network in delay nontolerant transmission mode with varying values of source transmission power $\left(P_{S}\right)$, where no. of $S D P s=10, d_{S_{m}, R}=d_{R, D_{n}}=1, \sigma_{R}^{2}=\sigma_{D}^{2}=$ 0.02 , and $\mathrm{B}=1 \mathrm{bps} / \mathrm{Hz}$.

also be observed that the linear energy harvesting models achieve better performance than nonlinear energy harvesting model because of the obvious reasons mentioned earlier.

Figures 9 and 10, respectively, plot the impact of sourceto-destination distance $d_{S_{m}, R}$ on overall network throughput for both delay tolerant and delay nontolerant transmission modes. The distance from relay to destination node $d_{R, D_{n}}$ is set to $d_{R, D_{n}}=2-d_{S_{m}, R}$. Values of other parameters are kept fixed; i.e., $S D P s=10, P_{S}=1$, and $\sigma_{R}^{2}=\sigma_{D}^{2}=0.02$.

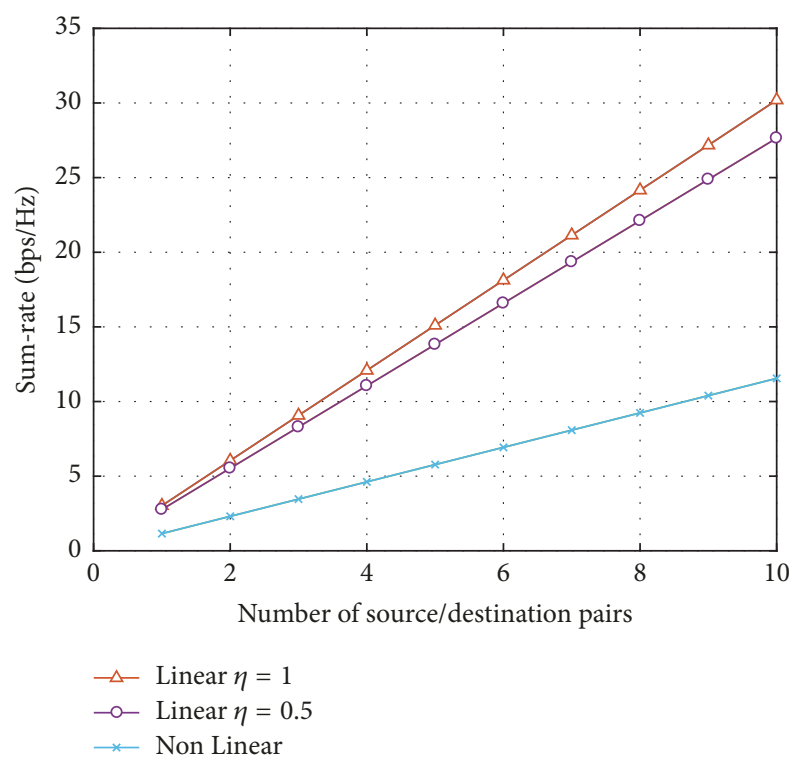

FIGURE 7: Network sum-rate of proposed network in delay tolerant transmission mode with varying number of source/destination pairs, where the values of other parameters are $d_{S_{m}, R}=d_{R, D_{n}}=1$, $P_{S}=1$, and $\sigma_{R}^{2}=\sigma_{D}^{2}=0.02$.

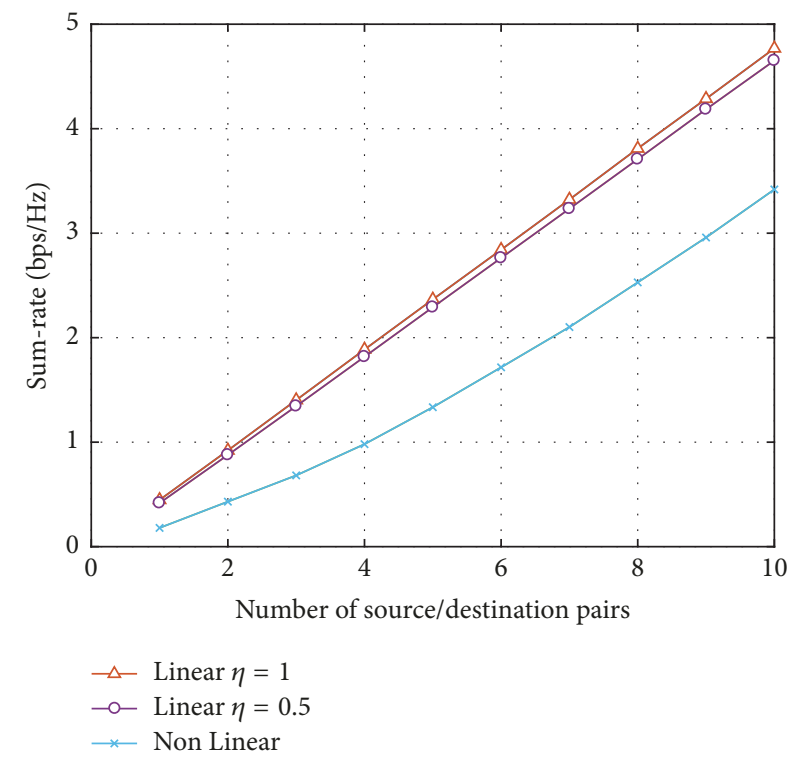

FIGURE 8: Network sum-rate of proposed network in delay nontolerant transmission mode with varying number of source/destination pairs, where the values of other parameters are $d_{S_{m}, R}=d_{R, D_{n}}=1$, $P_{S}=1, \sigma_{R}^{2}=\sigma_{D}^{2}=0.02$, and $\mathrm{B}=1 \mathrm{bps} / \mathrm{Hz}$.

It can be observed that in case of linear energy harvesting model the sum-rate of both transmission modes significantly decreases as the distance from source to relay $d_{S_{m}, R}$ increases. It is because received signal strength and harvested power (see (2)) at relay highly depends on the path loss $d_{S_{m}, R}^{-\alpha}$. On the other hand, in nonlinear energy harvesting model the sumrate starts increasing as the value of $d_{S_{m}, R}$ starts increasing. The reason for this is that due to the nonlinear behavior 


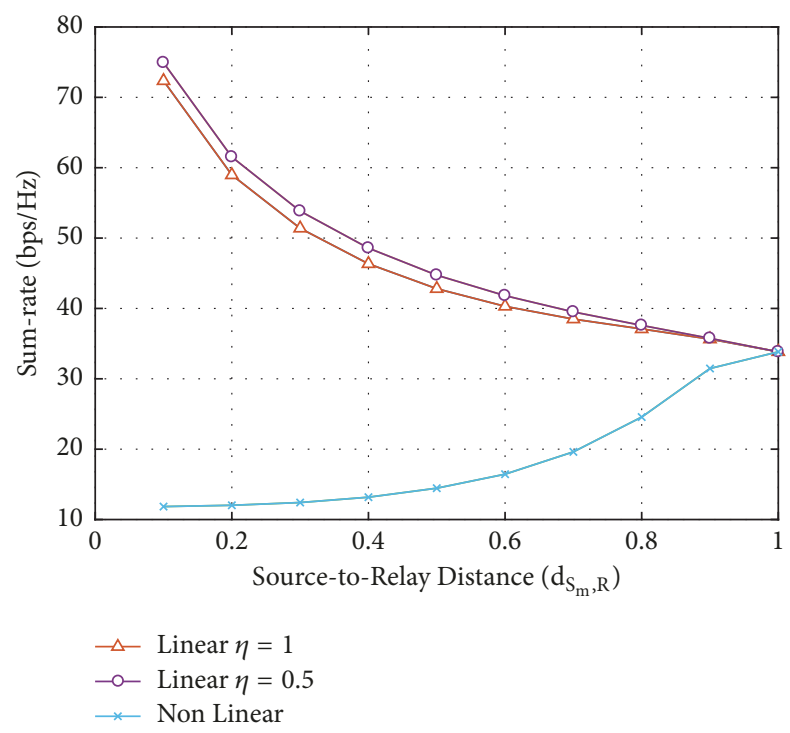

FIGURE 9: Network sum-rate of proposed network in delay tolerant transmission mode with varying values of source-to-relay distance $\left(d_{S_{m}, R}\right)$, where $d_{R, D_{n}}=2-d_{S_{m}, R}$, no. of SDPs $=10, P_{S}=1$, and $\sigma_{R}^{2}=\sigma_{D}^{2}=0.02$.

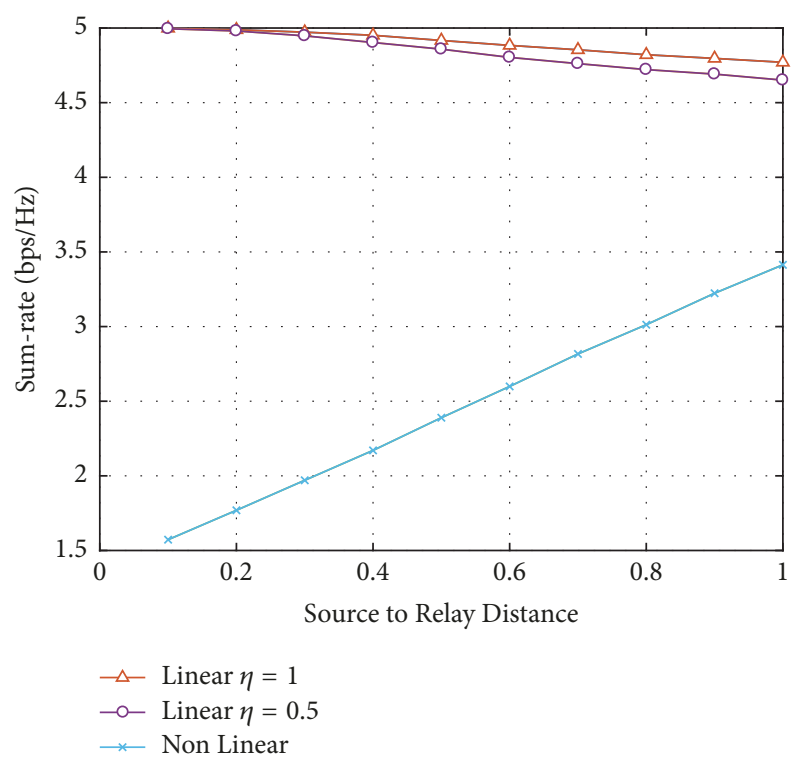

FIGURE 10: Network sum-rate of proposed network in delay nontolerant transmission mode with varying values of source-to-relay distance $\left(d_{S_{m}, R}\right)$, where $d_{R, D_{n}}=2-d_{S_{m}, R}$, no. of $S D P s=10, P_{S}=1$, $\sigma_{R}^{2}=\sigma_{D}^{2}=0.02$, and $\mathrm{B}=1 \mathrm{bps} / \mathrm{Hz}$.

of energy harvesting circuit, the shorter distance between the source and relay node might not necessarily result in higher harvested power. In addition to this harvested power constraint, the smaller distance between source and relay also results in the larger distance between relay and destination, which further reduces the received signal strength and SNR at the destination (see (13)). From the results in Figures 9 and 10 , it is important to note that the ideal location of a

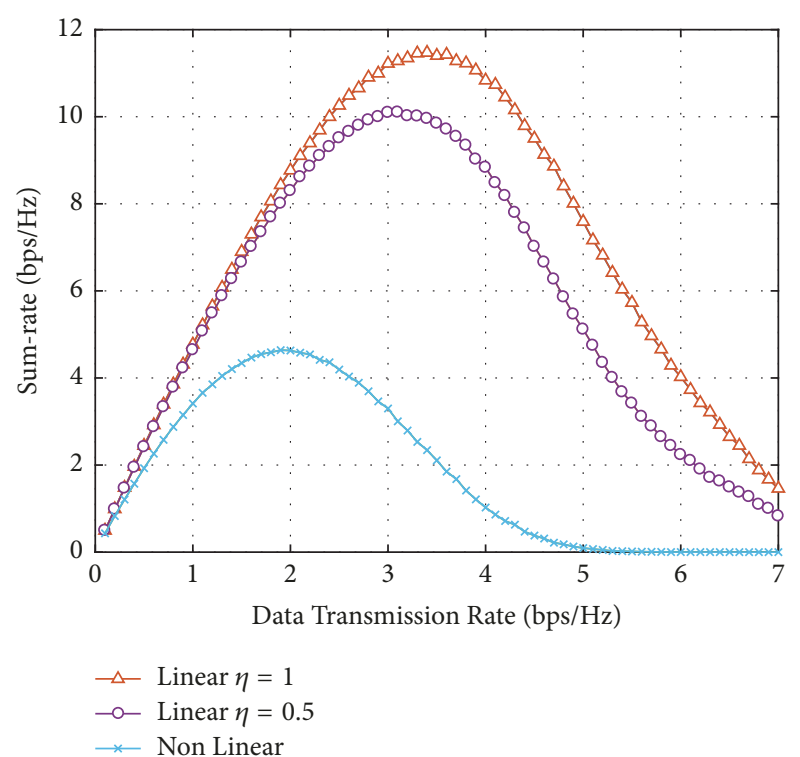

FIgURE 11: Achievable sum-rate of proposed scheme in delay nontolerant scheme with different values of source transmission rate (B), where other parameters are $d_{S_{m}, R}=d_{R, D_{n}}=1, P_{S}=1$, no. of $S D P s=10$, and $\sigma_{R}^{2}=\sigma_{D}^{2}=0.02$.

relay node in nonlinear energy harvesting network is not necessarily close to the source node. This is different from the impractical linear energy harvesting case [17] where locating the relay node close to source node improves the achievable throughput at the destination.

The impact of source transmission rate (B) on network sum-rate in delay nontolerant transmission mode is depicted in Figure 11. It can be observed from Figure 11 that the sumrate of both linear and nonlinear energy harvesting models increases as the transmission rate increases, but then after a certain point (i.e., $B \approx 3$ for linear and $B \approx 2$ for the nonlinear case) it starts declining. This is because the achievable throughput in delay nontolerant transmission mode highly depends on the transmission rate (see (17)) and therefore, at $B$ less than a certain value, the throughput at destination decreases. On the other hand, for the value of B larger than a certain value, the throughput again starts decreasing, because the destination node is unable to successfully decode the large amount of received data in limited time.

The effects of antenna noise variance at a destination node on overall system sum-rate of both delay tolerant and delay nontolerant transmission modes are depicted in Figures 12 and 13, respectively. It can be observed that the network sum-rate in all considered scenarios notably decreases as the antenna noise at destination increases. It is because the achievable throughput at destination nodes is highly affected by the antenna noise (see (13), (14), and (17)). Also, note that the effects of antenna noise variance on all considered scenarios are very similar, because the proposed scheme is using DF relay, which means that the overall sum-rate in every scenario is equally affected by the antenna noise variance. Figure 14 shows the total harvested power for 


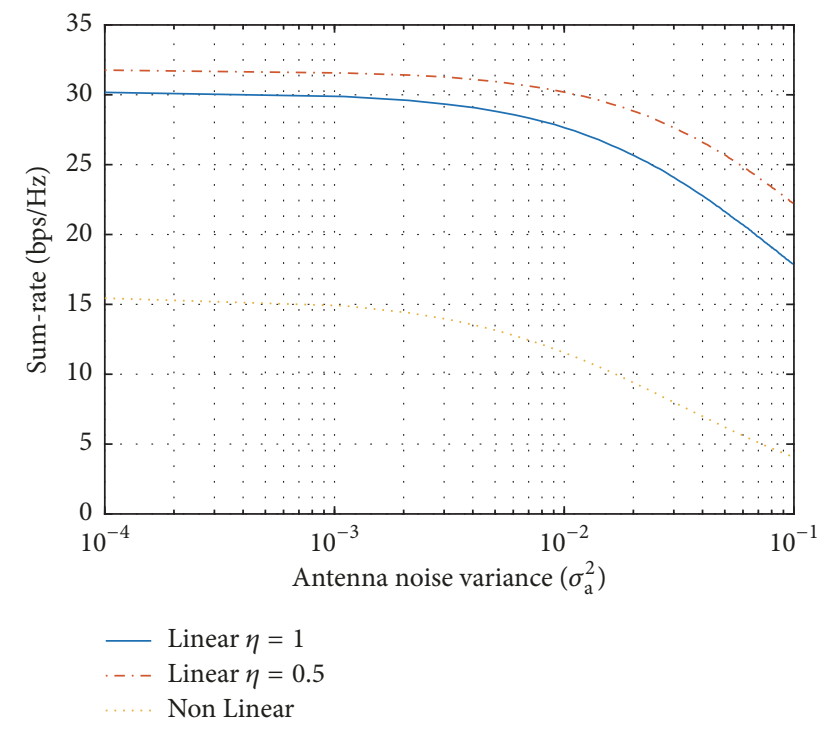

FIGURE 12: Network sum-rate of proposed network in delay tolerant transmission mode with different values of antenna noise, where other parameters are $d_{S_{m}, R}=d_{R, D_{n}}=1$, no. of $S D P s=10$, and $P_{S}=1$.

nonlinear delay tolerant scheme with varying values of source transmit power. It can be observed that the harvested power at relay node follows a nonlinear behavior and there is no significant change in the amount of harvested power after $P_{s}=0.15$. Furthermore, it can also be observed that the total amount of harvested power significantly increases as the number of SDPs increases. This is because more energy is harvested at relay node from increasing numbers of source signals power.

\section{Conclusion}

In this paper, a multipoint-to-multipoint energy harvestingbased relay network has been studied. In the proposed network, multiple source nodes communicate with their respective destination nodes via an intermediate energyconstrained (DF) relay. Two different source transmission modes, namely, delay tolerant and delay nontolerant transmission models, are studied. Efficient dynamic energy harvesting and power distribution schemes were developed and studied. In addition, for more practical and realistic analysis, we have considered a nonlinear energy harvesting model. With the help of numerical results, useful practical insights of our proposed schemes are provided. Furthermore, it has also been shown that compared to impractical linear energy harvesting model the practical nonlinear energy harvesting model exhibits different characteristics. As a future work, our proposed system architecture can be extended to a twoway relay network, where both communicating nodes act as a transceiver. Another interesting extension of this work would be the use amplify-and-forward relay with TSR or PSR protocols for energy harvesting.

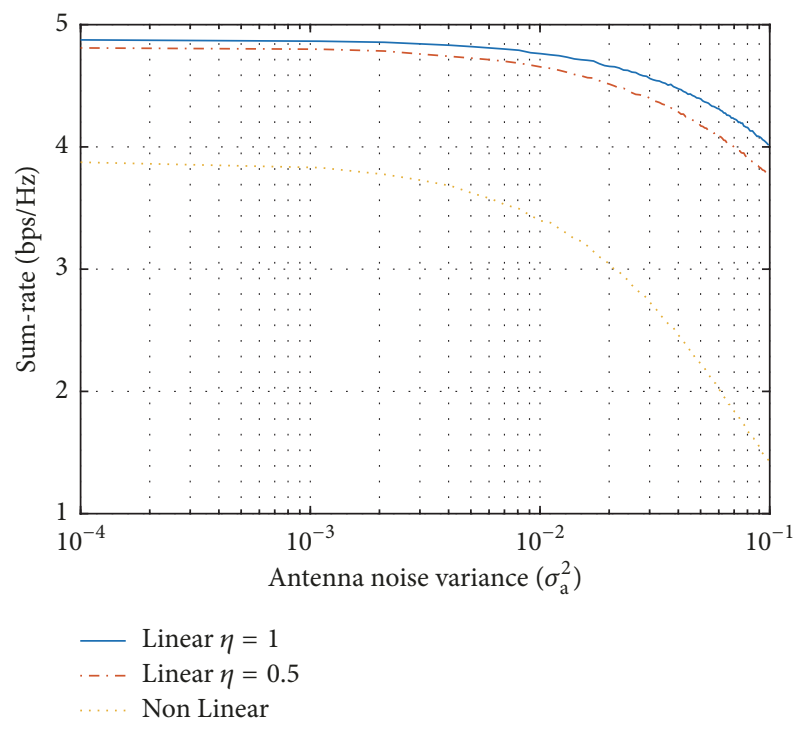

FIGURE 13: Network sum-rate of proposed network in delay nontolerant transmission mode with different values of antenna noise, where other parameters are $d_{S_{m}, R}=d_{R, D_{n}}=1$, no. of $S D P s=10$, $P_{S}=1$, and $B=1 b p s / H z$.

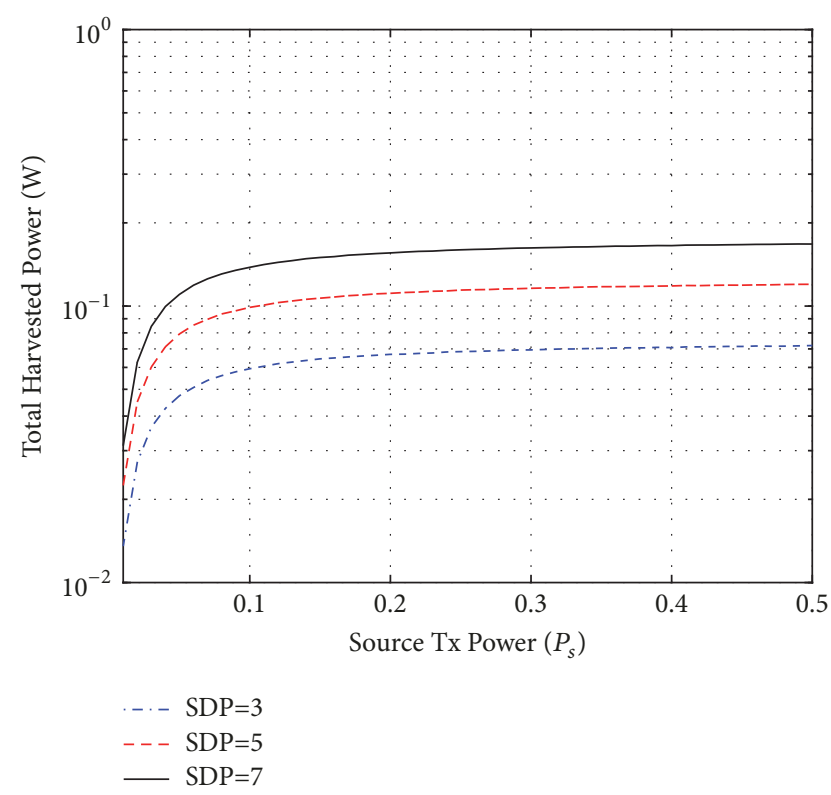

FIGURE 14: Total harvested power at relay node for nonlinear delay tolerant scheme with varying values of $P_{s}$ and SDPs.

\section{Data Availability}

The data included in this paper is solely obtained through computer simulations.

\section{Conflicts of Interest}

The authors declare that they have no conflicts of interest. 


\section{Acknowledgments}

This work was supported by the National Research Foundation of Korea (NRF) Grant funded by the Korean Government (MSIP) (2014R1A5A1011478).

\section{References}

[1] X. Lu, P. Wang, D. Niyato, D. I. Kim, and Z. Han, "Wireless networks with rf energy harvesting: a contemporary survey," IEEE Communications Surveys \& Tutorials, vol. 17, no. 2, pp. 757-789, 2015.

[2] L. R. Varshney, "Transporting information and energy simultaneously," in Proceedings of the IEEE International Symposium on Information Theory (ISIT '08), pp. 1612-1616, IEEE, Toronto, Canada, July 2008.

[3] D. S. Michalopoulos, H. A. Suraweera, and R. Schober, "Relay selection for simultaneous information transmission and wireless energy transfer: a tradeoff perspective," IEEE Journal on Selected Areas in Communications, vol. 33, no. 8, pp. 1578-1594, 2015.

[4] J. Xu and R. Zhang, "Throughput optimal policies for energy harvesting wireless transmitters with non-ideal circuit power," IEEE Journal on Selected Areas in Communications, vol. 32, no. 2, pp. 322-332, 2014.

[5] V. Raghunathan, S. Ganeriwal, and M. Srivastava, "Emerging techniques for long lived wireless sensor networks," IEEE Communications Magazine, vol. 44, no. 4, pp. 108-114, 2006.

[6] I. Ahmed, M. M. Butt, C. Psomas, A. Mohamed, I. Krikidis, and M. Guizani, "Survey on energy harvesting wireless communications: Challenges and opportunities for radio resource allocation," Computer Networks, vol. 88, pp. 234-248, 2015.

[7] R. Zhang and C. K. Ho, "MIMO broadcasting for simultaneous wireless information and power transfer," IEEE Transactions on Wireless Communications, vol. 12, no. 5, pp. 1989-2001, 2013.

[8] P. Popovski, A. M. Fouladgar, and O. Simeone, "Interactive joint transfer of energy and information," IEEE Transactions on Communications, vol. 61, no. 5, pp. 2086-2097, 2013.

[9] S. T. Shah, K. W. Choi, T. Lee, and M. Y. Chung, "Outage Probability and Throughput Analysis of SWIPT Enabled Cognitive Relay Network with Ambient Backscatter," IEEE Internet of Things Journal, 2018, https://ieeexplore.ieee.org/document/ 8360017/.

[10] E. E. Tsiropoulou, G. Mitsis, and S. Papavassiliou, "Interestaware energy collection \& resource management in machine to machine communications," Ad Hoc Networks, vol. 68, pp. 48-57, 2018.

[11] L. T. Dung, T. M. Hoang, N. T. Tan, and S. G. Choi, "Analysis of partial relay selection in NOMA systems with RF energy harvesting," in Proceedings of the 2018 2nd International Conference on Recent Advances in Signal Processing, Telecommunications \& Computing (SigTelCom), pp. 13-18, Ho Chi Minh City, Vietnam, January 2018.

[12] P. Vamvakas, E. E. Tsiropoulou, M. Vomvas, and S. Papavassiliou, "Adaptive power management in wireless powered communication networks: a user- centric approach," in Proceedings of the 2017 IEEE 38th Sarnoff Symposium, pp. 1-6, Newark, NJ, USA, September 2017.

[13] R. Pabst, B. H. Walke, D. C. Schultz et al., "Relay-based deployment concepts for wireless and mobile broadband radio," IEEE Communications Magazine, vol. 42, no. 9, pp. 80-89, 2004.
[14] H. Ding, D. B. Da Costa, H. A. Suraweera, and J. Ge, "Role Selection Cooperative Systems with Energy Harvesting Relays," IEEE Transactions on Wireless Communications, vol. 15, no. 6, pp. 4218-4233, 2016.

[15] K. W. Choi, L. Ginting, P. A. Rosyady, A. A. Aziz, and D. I. Kim, "Wireless-Powered Sensor Networks: How to Realize," IEEE Transactions on Wireless Communications, vol. 16, no. 1, pp. 221234, 2017

[16] X. Zhou, R. Zhang, and C. K. Ho, "Wireless information and power transfer: architecture design and rate-energy tradeoff," IEEE Transactions on Communications, vol. 61, no. 11, pp. 47544761, 2013.

[17] A. A. Nasir, X. Zhou, S. Durrani, and R. A. Kennedy, "Relaying protocols for wireless energy harvesting and information processing," IEEE Transactions on Wireless Communications, vol.12, no. 7, pp. 3622-3636, 2013.

[18] A. A. Nasir, X. Zhou, S. Durrani, and R. A. Kennedy, “Throughput and ergodic capacity of wireless energy harvesting based DF relaying network," in Proceedings of the 2014 1st IEEE International Conference on Communications, ICC '14, pp. 4066-4071, Australia, June 2014.

[19] D.-T. Do, "Time power switching based relaying protocol in energy harvesting mobile node: optimal throughput analysis," Mobile Information Systems, vol. 2015, Article ID 769286, 8 pages, 2015.

[20] S. T. Shah, D. Munir, M. Y. Chung, and K. W. Choi, "Information Processing and Wireless Energy Harvesting in Two-Way Amplify-and-Forward Relay Networks," in Proceedings of the 2016 IEEE 83rd Vehicular Technology Conference (VTC Spring), pp. 1-5, Nanjing, China, May 2016.

[21] S. T. Shah, K. W. Choi, S. F. Hasan, and M. Y. Chung, "Throughput analysis of two-way relay networks with wireless energy harvesting capabilities," Ad Hoc Networks, vol. 53, pp. 123-131, 2016.

[22] W. Xu, Z. Yang, Z. Ding, L. Wang, and P. Fan, "Wireless information and power transfer in two-way relaying network with non-coherent differential modulation," EURASIP Journal on Wireless Communications and Networking, vol. 131, pp. 1-10, 2015.

[23] S. T. Shah, K. W. Choi, S. F. Hasan, and M. Y. Chung, "Energy harvesting and information processing in two-way multiplicative relay networks," IEEE Electronics Letters, vol. 52, no. 9, pp. 751-753, 2016.

[24] Z. Ding, S. M. Perlaza, I. Esnaola, and H. V. Poor, "Power allocation strategies in energy harvesting wireless cooperative networks," IEEE Transactions on Wireless Communications, vol. 13, no. 2, pp. 846-860, 2014.

[25] I. Krikidis, S. Timotheou, and S. Sasaki, "RF energy transfer for cooperative networks: data relaying or energy harvesting?" IEEE Communications Letters, vol. 16, no. 11, pp. 1772-1775, 2012.

[26] M. Ashraf, J.-W. Jang, and K.-G. Lee, “Outage Probability Analysis for Energy Harvesting Cooperative Relays in a Clustered Environment," Mobile Networks and Applications, pp. 1-12, 2017.

[27] X. Liu, K. Chen, J. Yan, and Z. Na, “Optimal Energy Harvestingbased Weighed Cooperative Spectrum Sensing in Cognitive Radio Network," Mobile Networks and Applications, vol. 21, no. 6, pp. 908-919, 2016.

[28] P. He, L. Zhao, S. Zhou, and Z. S. Niu, Radio Resource Management Using Geometric Water-Filling, Springer-Verlag, New York, NY, USA, 2014.

[29] P. He, L. Zhao, S. Zhou, and Z. Niu, "Water-filling: A geometric approach and its application to solve generalized radio resource 
allocation problems," IEEE Transactions on Wireless Communications, vol. 12, no. 7, pp. 3637-3647, 2013.

[30] P. He and L. Zhao, "Optimal Power Allocation for CR MIMO Energy Harvesting Coexisting Systems," in Proceedings of the GLOBECOM 2015 - 2015 IEEE Global Communications Conference, pp. 1-6, San Diego, Calif, USA, December 2015.

[31] P. He and L. Zhao, "Noncommutative composite water-filling for energy harvesting and smart power grid hybrid system with peak power constraints," IEEE Transactions on Vehicular Technology, vol. 65, no. 4, pp. 2026-2037, 2016.

[32] J. Gu, T.-J. Lee, and M. Y. Chung, "Water-filling techniquebased proportionally fair scheduling algorithm for SC-FDMA," Transactions on Emerging Telecommunications Technologies, vol. 27, no. 12, pp. 1664-1671, 2016.

[33] S. Misra, S. D. Hong, G. Xue, and J. Tang, "Constrained relay node placement in wireless sensor networks: formulation and approximations," IEEE/ACM Transactions on Networking, vol. 18, no. 2, pp. 434-447, 2010.

[34] T. M. Hoang, N. T. Tan, N. B. Cao, and L. T. Dung, "Outage probability of MIMO relaying full-duplex system with wireless information and power transfer," in Proceedings of the 2017 Conference on Information and Communication Technology (CICT), pp. 1-6, Gwalior, India, November 2017.

[35] F. D'Andreagiovanni and A. Nardin, "Towards the fast and robust optimal design of wireless body area networks," Applied Soft Computing, vol. 37, pp. 971-982, 2015.

[36] J. Yick, B. Mukherjee, and D. Ghosal, "Wireless sensor network survey," Computer Networks, vol. 52, no. 12, pp. 2292-2330, 2008.

[37] S. Luo, R. Zhang, and T. J. Lim, "Optimal save-then-transmit protocol for energy harvesting wireless transmitters," IEEE Transactions on Wireless Communications, vol. 12, no. 3, pp. 1196-1207, 2013.

[38] C. K. Ho and R. Zhang, "Optimal energy allocation for wireless communications with energy harvesting constraints," IEEE Transactions on Signal Processing, vol. 60, no. 9, pp. 4808-4818, 2012.

[39] E. Boshkovska, D. W. K. Ng, N. Zlatanov, and R. Schober, "Practical non-linear energy harvesting model and resource allocation for SWIPT systems," IEEE Communications Letters, vol. 19, no. 12, pp. 2082-2085, 2015.

[40] M. O. Hasna and M.-S. Alouini, "Performance analysis of twohop relayed transmissions over Rayleigh fading channels," in Proceedings of the IEEE 56th Vehicular Technology Conference (VTC-Fall '02), vol. 4, pp. 1992-1996, Vancouver, Canada, 2002.

[41] B. Medepally and N. B. Mehta, "Voluntary energy harvesting relays and selection in cooperative wireless networks," IEEE Transactions on Wireless Communications, vol. 9, no. 11, pp. 3543-3553, 2010.

[42] T. Le, K. Mayaram, and T. Fiez, "Efficient far-field radio frequency energy harvesting for passively powered sensor networks," IEEE Journal of Solid-State Circuits, vol. 43, no. 5, pp. 1287-1302, 2008.

[43] A. J. Goldsmith and P. P. Varaiya, "Capacity of fading channels with channel side information," IEEE Transactions on Information Theory, vol. 43, no. 6, pp. 1986-1992, 1997.

[44] D. P. Palomar and J. R. Fonollosa, "Practical algorithms for a family of waterfilling solutions," IEEE Transactions on Signal Processing, vol. 53, no. 2, part 1, pp. 686-695, 2005.

[45] D. Bertsekas, Network Optimization: Continuous and Discrete Models, Athena Scientific, 1998.
[46] H. Meyr, M. Mseneclaey, and S. A. Fechtel, Digital Communication Receivers: Synchronization Channel Estimation and Signal Processing, John Wiley \& Sons, Inc., 1998. 


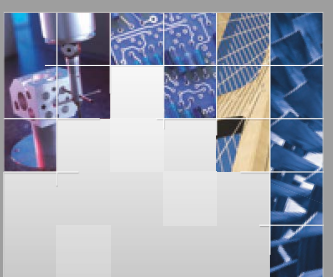

\section{Enfincering}
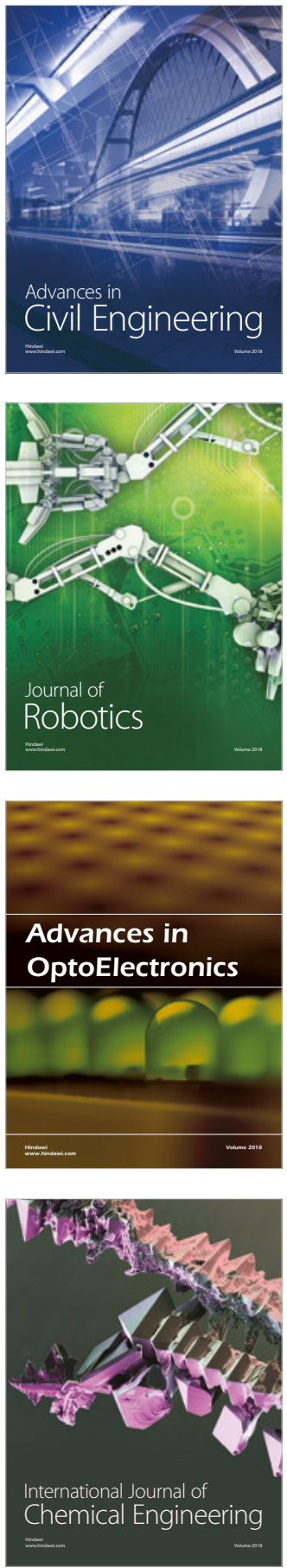

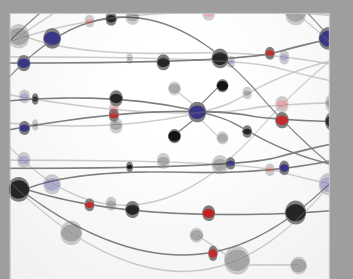

\section{Rotating \\ Machinery}

The Scientific World Journal

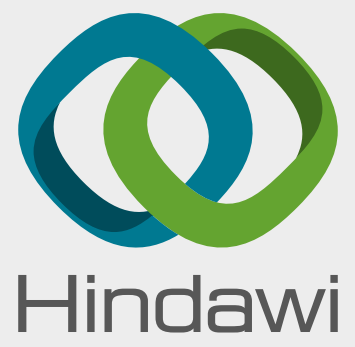

Submit your manuscripts at

www.hindawi.com
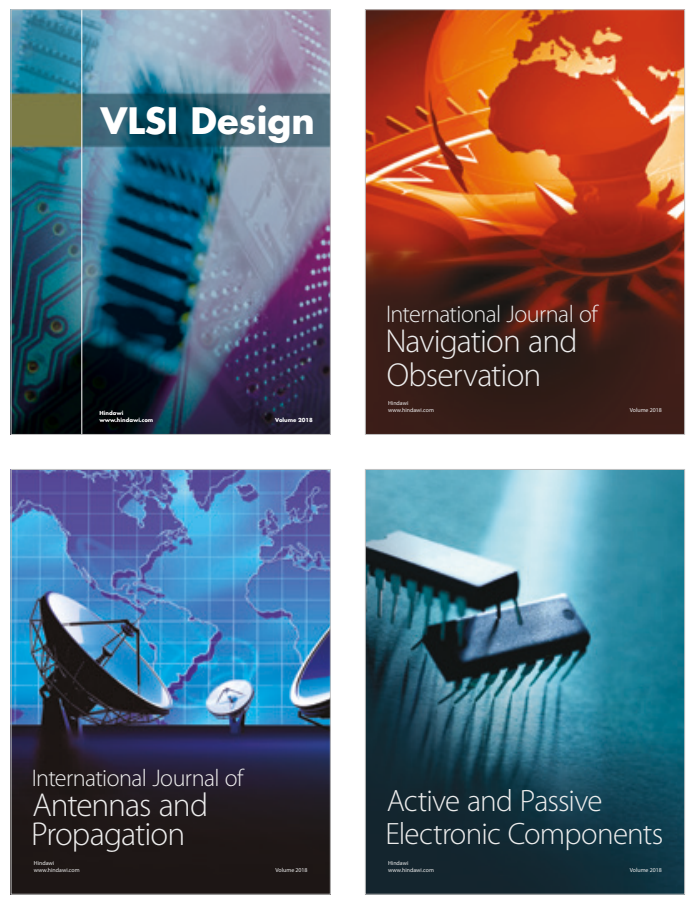
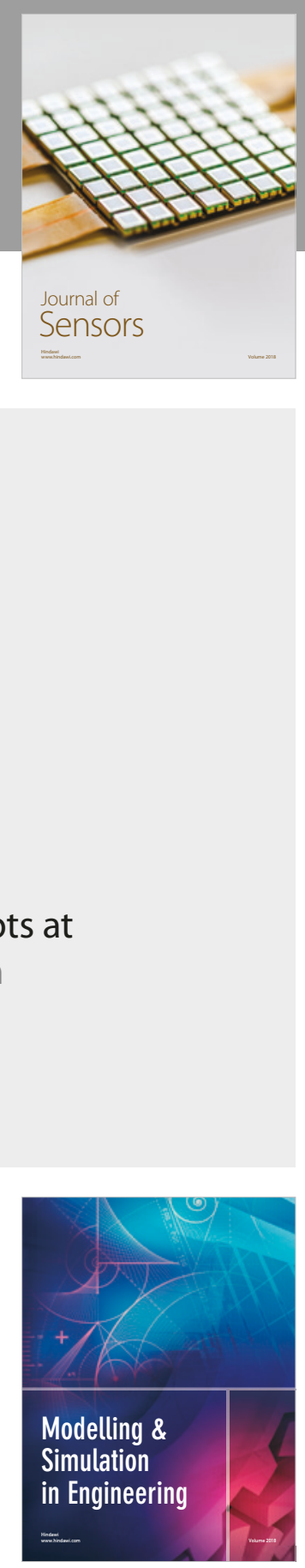

\section{Advances \\ Multimedia}
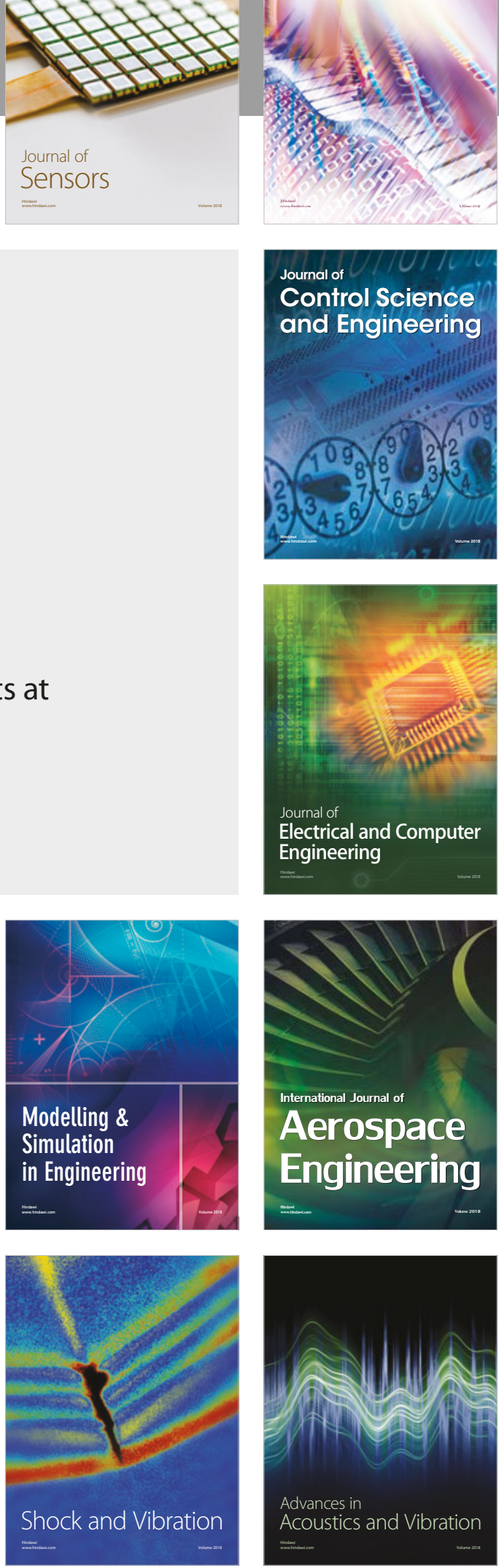